\title{
Diseases of Children
}

In 72 of these a growth was obtained, and in the remaining 275 the result was negative. The number of cases was too small to allow any decision to be made as to the relative value of the different methods of disinfection used, but Steinegger claims that the general result clearly shows that a knife used for a skin incision should be regarded as a possible source of infection if used for the deeper layers of a wound. The organism most commonly found was the staphylococcus albus.

J. W. S.

\section{DISEASES OF CHILDREN.}

UNDER THE CHARGE OF

A. H. MElville DUNLOP, M.D., AND A. DINGWALL FORDYCE, M.D.

The Binet-Simon Tests.

In order to ascertain the mental level of the child, and to discriminate between the child who is educationally backward but of normal intelligence, and the child whose intelligence is below the normal, the tests recommended by Binet and Simon have been used in Summerton School for mentally defective children (Fraser, School Hygiene, May, 1913).

According to the Defective and Epileptic Children (Education) Act, those children are to be admitted to special classes "who, not heing imbecile, and not merely dull and backward, are defective, that is to say, by reason of mental (or physical) defect are incapable of receiving proper benefit from the instruction in the ordinary public elementary schools, but are not incapable by reason of such defect of receiving benefit from instruction in special classes."

The difficulty is to decide which are these children.

Children who are making no progress in an ordinary school are not necessarily mentally defective; their backwardness may be due to a variety of causes, e.g., physical defects (slight deafness, visual defects, presence of adenoids and enlarged tonsils), irregular attendance, want of sufficient sleep, too high a class, etc.

Any physical defect must be remedied before judgment is passed on the mental condition of the child.

Binet and Simon, experimenting with a large number of normal children attending the elementary schools in Paris, found that at certain ages the intelligence of the child had so developed that he could then perform certain acts and answer certain questions. As a result of these extensive experiments a table was drawn up for the determination of the intellectual level of the child according to certain specified age-periods.

In a number of cases it will be found that some of the tests cannot be applied at all. The value of the tests is greatest in determining the intellectual level in children apparently normal till sent to school. 
These are the most important cases for admission to the special school, since some hope may be entertained of improvement under special instruction. It is advisable to begin with the tests for an age lower than that of the child, because if too difficult a question is set or asked the child becomes frightened and no further response can be elicited.

A consideration of some of the more important tests and of the results arrived at when applied to defective children in Summerton Special School is of interest.

Description of Pictures.-Various pictures are shown to the child, who is asked to tell what he sees. By means of this test Binet distinguishes three intellectual levels:-

1. At 3 years the normal child enumerates the objects seen, e.g. a man, a dog, a hat, a stick. This shows a simple perception and recognition of external objects.

2. Later (at 7 years), the character of the persons and the nature of the things are described and their connections noted. This indicates a power of description and association.

3. Still later the child interprets the picture and gives some evidence of his reasoning power.

In Summerton School no child under 9 years (with one exception of 7 years) described the pictures, that is to say, they were two or more years behind the normal child by this test. Of those over 9 who described the pictures, half described them accurately. Three children aged 10, 11, and 12 years respectively described and interpreted the pictures.

By these tests valuable information is obtained regarding the children's powers of perception, observation, and interpretation. Generally it is found that in these respects the defective children in schools are more than two years behind normal children.

Definition of Known Objects.-The objects to be defined are fork, horse, chair, mother. The child is asked, "What is a fork?" etc. This shows the child's power of expression, and elucidates the conception he has formed of an object, and the point of view which he considers to be the most important. Binet describes three classes of answers: (1) Silence, or mere repetition of the word ; (2) definition of use ( 7 years); (3) definition superior to use (9 years).

Memory is tested in various ways.

The auditory memory is tested by reading the following passage to the child :- " Last night, in Glasgow, a fire destroyed three buildings in the centre of the town. Seventeen families are without homes. In saving a baby, a boy got his hands badly burnt."

In using this test Binet makes the normal child read the passage, but in dealing with defective children this is impossible, as they can rarely read. After reading it to the child he is asked what the passage is about. He is told beforehand to listen carefully, as he will have to 
remember it. At eight years he should be able to remember two facts.

This test does not give satisfactory results in testing the mentally deficient, as it depends to a large extent on attention and concentration as well as on memory-powers in which the mentally defective child is notably deficient. Dr. Shrubsall considers it too hard even for mentally defectives who are leaving school. Three of the Summerton cases remembered two facts, aged 9,11 , and 12 respectively. One boy of 13 remembered the whole passage.

In many cases where the facts were not remembered something was invented to take their place. The normal child who does not remember says so or remains silent.

The immediate memory for unrelated auditory stimuli is tested by repeating numbers. This involves an effort of attention, as the isolated figures convey no meaning.

A normal child should repeat 3 numbers at 4 years, 5 numbers at 7 years, 7 numbers at 12 years.

Care must be taken that the figures used are the same in every case, otherwise the results are not comparable.

The immediate memory for intimately associated auditory stimuli is tested by repeating phrases. Normal children of 6 years old should be able to repeat a phrase of sixteen syllables.

Binet, comparing twelve defectives aged 9 years with twelve normal children of the same age, found the defectives very feeble in this respect. He found one or two exceptions, but on closer examination he thought the ability of those to repeat was an "echo," and that if an interval was allowed these also failed in the test.

Judgment of Length.--This is determined by means of a card on which are drawn two lines, one $5 \mathrm{~cm}$. and the other $6 \mathrm{~cm}$. long, placed $3 \mathrm{~cm}$. apart. The card is shown to the child with the longer line placed to the right, to the left, above and below the shorter, and in each position the child is asked to point to the longest. Suggestibility is introduced by immediately afterwards showing two lines of exactly the same length.

The normal child of 4 should accomplish this test. Dr. Shrubsall says that mentally deficients pass this test. Binet considers that the response to the test shows little difference between the normal and the defective child. Whipple says: "Defectives are apt to answer fairly promptly, but repeated trials may show that they are not really comparing the lines."

The author compared the results obtained by this test with twenty children in an ordinary school and twenty children in a defective school, the ages ranging from 7 to 14 years. Of the twenty children in the ordinary school eighteen promptly pointed to the longer line. Only one, a boy aged 14 years, said that the lines of equal length were 
the same. The other nineteen all pointed to one or other line as being the longer, but in each case after considerable hesitation. It seems probable that they considered the lines to be the same, but felt they were expected to find a difference. Of the twenty children in the defective school thirteen answered correctly as to the longest line, while every child pointed to one or other of the lines of equal length, and that without any hesitation.

The Comparison of. Weights.-For this test four small boxes of the same size and shape, and weighing 45 grs., 3 i. ss., jiii., and jiv. respectively, are placed before the child, who is then asked to put the heaviest in a certain spot indicated, the next heaviest next, and so on. This is much more difficult than simply placing the different weights in the hand and asking which is the heaviest, for not only is an appreciation of the sense of weight required, but also the understanding that it is necessary to compare the weights in order to put them in proper order.

Binet finds that two weights can be compared at 5 years, while five weights can be arranged at 9 years.

In the mentally defective the sense of weight is often feeble, and it is impossible to get them to arrange the weights in the order required.

Comparison of Two Objects from Memory.-The objects compared are paper and cardboard, fly and butterfly, wood and glass.

At 8 years the normal child can compare objects, and does so readily. It is often difficult to make the mentally defective child understand what is wanted. He will say, for example, that wood and glass are not the same, but when asked what is different about them will say: "Wood's different," or "glass is different." He apparently knows the difference, but cannot express it.

Tracing Resemblance-Tracing resemblance between two objects is still more difficult, and very few of the children succeeded in doing so. They frequently gave the contrast instead of the similarity.

The words used are snow and milk, fly and bee, table, chair, door. If a child responds to this test his deficiency may be doubted.

Framing Sentences.-Framing a sentence containing three given words is a more difficult test, and requires some inventive power. The words used are girl, river, ball.

The normal child can do this at 10 years.

Recognising Wants or Deficiencies in Figures._Three faces are shown -one wanting eyes, one wanting nose, one wanting mouth, and a figure of a woman is shown without arms.

Here there is a marked difference between the normal and the abnormal child. The normal child recognises them promptly by 7 years.

The author has only seen one defective child (aged 12 years) recognise the wants at once without help.

Execution of Three Simultaneous Orders.-This shows response, and 
Binet finds that the normal child carries out the three orders promptly by 6 years. Other observers find it done earlier by the normal child, while Binet puts it at 7 years in his 1911 series. Most defectives fail to carry out more than two commands.

Colour Sense.-The colour sense is tested both by naming and match-

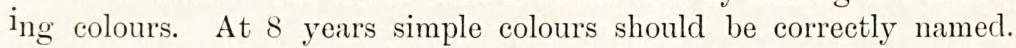
Colour defect does not necessarily mean mental deficiency. The defective child can often match the colours though unable to name them.

Many of the other tests, such as giving the date, counting pennies, giving change, etc., involve a certain amount of instruction, and consequently are not so valuable as indicators of intelligence, though of use in determining how much the child has learned.

Questions of Comprehension.-The questions of comprehension are, however, very valuable as indicating the mental condition of the child.

Binet has arranged these in two series, one more difficult and presenting more difficulties in vocabulary. The first, series are usually answered at 10 years by the normal child.

The defective child answers simple but fails with abstract questions, and the answers themselves give indications as to the child's tendencies, habits, and mental outlook.

The dependence of such children is shown by the frequency with which they say they would tell their mother; while their timidity is expressed by the number of times the questions are answered by the word "greet" (cry).

The results of systematic application of these tests to defective children by Decroly and Degard show-" That there is a very good general correspondence between the classification of the defectives on the basis of pedagogical, clinical, and general observation, and their classification on the basis of the test, so that the tests have a practical value for the psyciological classification of defectives."

One objection lodged against the system is the length of time occupied in testing each child. Binet gives the time as forty minutes, but it is rarely possible to examine the defective child fully in less than anl hour.

It may be reasonably asserted that the time occupied is a saving of time and a preventive of mistakes in the future, because if the tendencies and characteristics of the child are known on his entrance to the class, the scope and extent of his education will be determined. 